\title{
Intraoperative burst suppression is associated with postoperative delirium following cardiac surgery: a prospective, observational study
}

Martin Soehle ${ }^{1 *+}$, Alexander Dittmann ${ }^{2 \dagger}$, Richard K Ellerkmann ${ }^{1}$, Georg Baumgarten ${ }^{1}$, Christian Putensen ${ }^{1}$ and UIf Guenther ${ }^{1}$

\begin{abstract}
Background: Postoperative delirium (POD) occurs frequently after cardiac surgery and is associated with increased morbidity and mortality. We analysed whether perioperative bilateral BIS monitoring may detect abnormalities before the onset of POD in cardiac surgery patients.

Methods: In a prospective observational study, 81 patients undergoing cardiac surgery were included. Bilateral Bispectral Index (BIS)-monitoring was applied during the pre-, intra- and postoperative period, and BIS, EEG Asymmetry (ASYM), and Burst Suppression Ratio (BSR) were recorded. POD was diagnosed according to the Confusion Assessment Method for the Intensive Care Unit, and patients were divided into a delirium and non-delirium group.

Results: POD was detected in 26 patients (32\%). A trend towards a lower ASYM was observed in the delirium group as compared to the non-delirium group on the preoperative day (ASYM $=48.2 \pm 3.6 \%$ versus $50.0 \pm 4.7 \%$, mean \pm sd, $p=0.087$ ) as well as before induction of anaesthesia, with oral midazolam anxiolysis (median ASYM $=49.5 \%, I Q R$ [47.4;51.5] versus $50.6 \%, I Q R ~[49.1 ; 54.2], p=0.081)$. Delirious patients remained significantly $(p=0.018)$ longer in a burst suppression state intraoperatively (107 minutes, IQR [47;170] versus 44 minutes, IQR [11;120]) than non-delirious patients. Receiver operating analysis revealed burst suppression duration (area under the curve $=0.73, p=0.001$ ) and BSR ( $A \cup C=0.68, p=0.009)$ as predictors of POD.
\end{abstract}

Conclusions: Intraoperative assessment of BSR may identify patients at risk of POD and should be investigated in further studies. So far it remains unknown whether there is a causal relationship or rather an association between intraoperative burst suppression and the development of POD.

Trial registration: clinicaltrials.gov NCT01048775

Keywords: Cardiac surgery, Postoperative delirium, Outcome, Electroencephalogram, Burst suppression, Bispectral Index

\section{Background}

Delirium is defined as an acute disturbance of consciousness with a fluctuating course that affects attention, cognition, emotionality, and the sleep-wake cycle [1]. Following cardiac surgery using extracorporeal circulation, postoperative delirium (POD) occurs frequently with a reported incidence ranging between 14 and $51 \%$ [2-5]. It is associated with a prolonged stay in the intensive care unit, as well as

\footnotetext{
* Correspondence: martin.soehle@ukb.uni-bonn.de

${ }^{\dagger}$ Equal contributors

'Department of Anaesthesiology and Intensive Care Medicine, University of Bonn, Bonn, Germany

Full list of author information is available at the end of the article
}

an increased morbidity and mortality [5-7]. Hence, the success of cardiac surgery is seriously imperiled by the development of POD, and measures to predict and prevent POD are urgently sought.

The electroencephalogram (EEG) has been shown to be affected by POD in terms of an increased delta- and thetaas well as a decreased alpha- and beta-activity [8-10]. The main generators of this delirium-related theta increase have been reported to be localized in the anterior cingulate cortex as well as the right fronto-temporal brain areas [11]. A classic multi-lead EEG, recorded from the full set of scalp electrodes, is impractical in the acute clinical setting of anaesthesia and impossible to be analysed online 
by the untrained anaesthetist. Processed EEG monitors, such as the Bispectral Index (BIS) monitor, record one or two EEG channels only, and transform the complex EEG information into a few quantitative numbers, $[12,13]$ such as the BIS, the EEG Asymmetry (ASYM) or the Burst Suppression Ratio (BSR). Even though the BIS monitor has been developed to quantify depth of anaesthesia (and not the degree of delirium), it might nevertheless provide useful clinical information to identify patients at risk for POD or even predict POD. With the increased EEG activity in right frontal-temporal brain areas during POD, [11] our hypothesis was that patients with postoperative delirium will have EEG asymmetry not seen in non-delirious patients. This may occur at any time in the perioperative course. POD as well as the occurence of EEG burst supression has been shown independantly to be associated with an increased mortality, [14,15] however the relation between burst suppression and POD has remained unclear. We hypothesized that patients with postoperative delirium will spend more time in burst suppression and exhibit a higher burst suppression rate than nondelirious patients. We therefore performed a prospective study to test these hypotheses.

\section{Methods}

This study has been performed in accordance with the Declaration of Helsinki and has been approved by an appropriate ethics committee (Ethik-Kommission der Medizinischen Fakultät, Rheinische Friedrich-Wilhelms-Universität, Bonn, Germany; Approval No. 056 / 09). With written informed consent, we performed a prospective observational study, which was registered at ClinicalTrials.gov (NCT 01048775). Patients with an age of at least 60 years who were scheduled for elective cardiac surgery were included in the study. Exclusion criteria were pregnancy, emergency or off-pump cardiac surgery.

\section{BIS monitoring}

Bilateral BIS monitoring was applied to the patient's forehead as recommended by the manufacturer and connected to a BIS VISTA Monitor (version: platform 2.03, application 3.00, hardware revision 3.00). Data were sampled in 5-second intervals for later analysis. Three parameters were analyzed offline: BIS, ASYM and BSR. The BIS quantifies the depth of anaesthesia in a range between 100 (fully awake patient) and 0 (isoelectric EEG) [16]. ASYM denotes the asymmetry in the EEG total power (within the frequency range from $0-30 \mathrm{~Hz}$ ) comparing the left and right hemisphere, according to the formula [17]:

$$
A S Y M=(\text { total power left } / \text { total power left }+ \text { right }) * 100 \%
$$

Hence, an ASYM of 50\% represents equal total power in both hemispheres, whereas an ASYM $<50 \%$ indicates less total power in the left than the right hemisphere. The Burst
Suppression Ratio (BSR) is defined as the percentage of epochs in the previous 63 seconds in which the EEG signal is considered suppressed [18]. Suppression is recognized as those periods longer than 0.5 seconds, during which the EEG voltage does not exceed approximately $\pm 5 \mu \mathrm{V}[18,19]$.

In the preoperative period, the bilateral BIS was monitored in a calm surrounding on the afternoon prior to surgery. To do so, patients were asked to lie down on their bed, to close their eyes, and to relax during the BIS recording, which was performed by the same investigator (AD) over a period of 20 minutes in a standardized manner. On the following day, the bilateral BIS was recorded from entering the induction room during the entire surgery (intraoperative period). The EEG in the period from induction until onset of cardiopulmonary bypass (CPB) was analysed separately since this epoch was considered to be unaffected by any inflammatory process triggered by $\mathrm{CPB}$. Bilateral BIS monitoring was continued during the intensive care unit (ICU) stay (postoperative period) until discharge. The same BIS sensor was used for the entire period, unless an insufficient adhesion required a sensor replacement. During cardiac surgery, anaesthetists were blinded to the BIS and asked to perform anaesthesia according to clinical routine.

\section{Anaesthesia}

Oral midazolam $(7.5 \mathrm{mg})$ was administered one hour before induction of anaesthesia. Anaesthesia was induced with etomidate ( $0.2 \mathrm{mg} / \mathrm{kg}$ b.w.), sufentanil $(10 \mu \mathrm{g} / \mathrm{kg} / \mathrm{h}$ until a total dose of $\sim 0.5 \mu \mathrm{g} / \mathrm{kg}$ b.w. was reached) and cis-atracurium $(0.15 \mathrm{mg} / \mathrm{kg}$ b.w.). The trachea was intubated and anaesthesia was maintained with isoflurane ( 0.7 MAC), sufentanil $(\sim 1 \mu \mathrm{g} / \mathrm{kg} / \mathrm{h})$ and cis-atracurium. Patients were transferred from the induction room to the adjacent operating room at the discretion of the attending anaesthesiologist. During $\mathrm{CPB}$, isoflurane was directly applied to the extracorporeal oxygenator and monitored using the gas analyzer of the anaesthesia ventilator. Following surgery, all patients were transferred to ICU, where patients were sedated with continuous propofol and sufentil according to our institution's standard operating procedure. Sedation was stopped as soon as the patients were in a stable condition.

\section{Monitoring for delirium and outcome}

Postoperatively, patients were monitored for the occurrence of POD using the flowsheet version of the Confusion Assessment Method for Intensive Care Units (CAM-ICUFlowsheet), which is explained in more detail elsewhere $[20,21]$. The CAM-ICU was performed once every day by the same investigator (AD). According to CAM-ICU criteria, $[20,21]$ patients were rated as having POD if they were CAM-ICU positive for at least a single examination. Depending on their Richmond Agitation-Sedation Score (RASS), [22] delirious patients were subgrouped as either hypoactive $($ RASS $\leq 0)$ or hyperactive $($ RASS $>0$ ). Patients 
that alternated between positive and negative RAS scores were grouped as having a mixed delirium [23]. Activity of Daily Living was assessed on the day prior to surgery as well as 6 months after: To do so, a questionnaire was completed in which the "Alzheimer's Disease Cooperative Study Mild Cognitive Impairment Activities of Daily Living Scale" (ADCS-MCI-ADL) was determined, [24] which ranges from 0 (completely depending on support) to 57 (totally independent). Mortality was assessed at 6 months after surgery.

\section{Statistics}

Patients were divided into a group with and without delirium, and data were averaged within groups. Data are shown as mean \pm standard deviation in case of normal distribution or otherwise as median and interquartile range. Groups were compared by $t$-test or Mann-Whitney rank sum test, respectively. Receiver operating characteristic (ROC) analysis was performed to investigate the sensitivity, specificity and predictive value of certain parameters to predict POD. ROC analysis and statistical tests were performed using SigmaPlot-Software (version 12.3, Systat Software Gmbh, Erkrath, Germany). Statistical significance was assumed at $\mathrm{p}<0.05$.

\section{Results}

\section{Epidemiology}

Eighty-seven patients $(n=87)$ were included into the study, four of whom died during their intensive care stay (perioperative mortality rate $=4.6 \%$ ). These were excluded since they could not be screened for the occurrence of POD. Another two patients were excluded: One withdrew consent, the other was unintentionally not intraoperatively monitored. Therefore, eighty-one patients $(\mathrm{n}=81)$ remained for analysis:

Patients consisted of 24 women and 57 men with a mean age of $72.9 \pm 6.2$ years, a mean height of $171.3 \pm$ $8.5 \mathrm{~cm}$ and a median weight of $76[70 ; 88] \mathrm{kg}$ (Table 1 ).
Coronary artery bypass grafting (CABG) was performed in 44 , valve replacement or reconstruction in 22 , and a combined CABG/valve-surgery in 15 patients. Surgery lasted for $5.4 \pm 1.2$ hours, of which $2.2 \pm 0.6$ hours were spent in $\mathrm{CPB}$ and $1.5 \pm 0.5$ hours in aortic clamping.

\section{Postoperative delirium}

Twenty-six patients (32\%) developed POD. Patients with and without POD did not differ statistically with respect to age, gender, height and type of surgery (Table 1). However patients with delirium tended to have a (non significantly) higher age $(74.5 \pm 6.5$ years, $\mathrm{p}=0.10)$ as well as a (non significantly) lower body weight (median of $74[69 ; 78] \mathrm{kg}, \mathrm{p}=0.08)$ as compared to non-delirious patients (age $=72.1 \pm 5.9$ years, weight $=80[70 ; 92] \mathrm{kg}$, Table 1). No statistical difference was observed between patients with or without delirium with respect to comorbidities, preoperative serum electrolytes and intraoperative parameters, such as arterial blood pressure, body temperature or the amount of anaesthetic drugs administered (Tables 2 and 3). ROC analysis for age yielded an area under the curve of 0.61 , which was statistically not significant $(\mathrm{p}=0.10$, Figure 1 , Table 4$)$. Hence, age was not a predictor of POD in our study.

The hypoactive subtype was observed as the dominant type of delirium, which occurred in 20 patients (77\%). The mixed and the hyperactive subtype were diagnosed in 5 and 1 patient, respectively. Patients with delirium spent almost double the time in ICU (median $=81$ [23; 141] hours) as compared to non-delirious patients (median $=42$ [20; 90] hours, $\mathrm{p}=0.033$ ).

The intraoperative BIS did not differ between patients who later developed POD (BIS $=44.6 \pm 5.5)$ and those who never developed POD (BIS $=45.1 \pm 7.7$ ).

Preoperative and 6-month ADCS-MCI-ADL scores did not differ between surviving patients with postoperative delirium and those who were not delirious. At 6-months, a significantly $(\mathrm{p}=0.03)$ higher mortality was observed in

Table 1 Patient characteristics

\begin{tabular}{|c|c|c|c|c|c|}
\hline \multirow{2}{*}{ Group } & & \multirow[b]{2}{*}{ All } & \multicolumn{2}{|l|}{ Patients } & \multirow[t]{2}{*}{$\mathrm{p}$-value } \\
\hline & & & With delirium & Without delirium & \\
\hline Number & & 81 & 26 & 55 & \\
\hline Age & [years] & $72.9 \pm 6.2$ & $74.5 \pm 6.5$ & $72.1 \pm 5.9$ & 0.101 \\
\hline Gender & {$[\mathrm{m} / \mathrm{f}]$} & $57 / 24$ & $16 / 10$ & $41 / 14$ & 0.299 \\
\hline Height & {$[\mathrm{cm}]$} & $171.3 \pm 8.5$ & $169.6 \pm 8.2$ & $172.2 \pm 8.6$ & 0.212 \\
\hline Weight & {$[\mathrm{kg}]$} & $76.0[70.0 ; 88.0]$ & $74.0[69.3 ; 78.3]$ & 80.0 [70.0; 92.0] & 0.083 \\
\hline \multicolumn{6}{|l|}{ Surgery: } \\
\hline & CABG & 44 & 13 & 31 & 0.638 \\
\hline & Valve & 22 & 8 & 14 & 0.605 \\
\hline & Misc & 15 & 5 & 10 & 1.000 \\
\hline
\end{tabular}

Data are displayed as mean \pm std dev in case of normal distribution or otherwise as median and interquartile range. CABG $=$ coronary artery bypass grafting. Statistic analysis revealed no significant differences in the above mentioned parameters between patients with and without delirium 
Table 2 Comorbidity and plasma electrolyte concentrations in comparison between delirious and non-delirious patients

\begin{tabular}{|c|c|c|c|c|}
\hline & & Patients with delirium $(n=26)$ & Patients without delirium $(n=55)$ & p-value \\
\hline \multicolumn{5}{|l|}{ Comorbidity } \\
\hline Congestive heart failure & & 9 & 24 & 0.48 \\
\hline Myocardial infarction & & 6 & 18 & 0.44 \\
\hline Diabetes mellitus & & 7 & 15 & 1.00 \\
\hline COPD & & 5 & 8 & 0.75 \\
\hline Peripheral vascular disease & & 2 & 5 & 1.00 \\
\hline Cerebrovascular disease & & 3 & 4 & 0.20 \\
\hline \multicolumn{5}{|c|}{ Preoperative plasma electrolyte concentrations } \\
\hline Sodium & {$[\mathrm{mmol} / \mathrm{l}]$} & $140[137.8 ; 142.3]$ & $140[139 ; 141]$ & 0.83 \\
\hline Potassium & {$[\mathrm{mmol} / \mathrm{l}]$} & $3.7[3.6 ; 4.1]$ & $3.8[3.5 ; 4.1]$ & 0.96 \\
\hline
\end{tabular}

The number of patients with certain comorbities is shown in the upper part. COPD = chronic obstructive pulmonary disease. Electrolyte concentrations are expressed as medians and interquartile range. Groups did not differ significantly with respect to the above shown parameters.

the delirium group (11.5\%) as compared with the nondelirium group (0\%).

\section{EEG asymmetry}

ASYM increased (non-significantly) from $49.4 \pm 4.5 \%$ on the previous day to $50.7 \pm 4.1 \%$ prior to induction. Apart from this initial rise, ASYM was approximately $49 \pm 3 \%$ for most of the remaining time, indicating a slightly higher total EEG power over the right hemisphere. Patients who later on developed POD showed a nonsignificant trend towards a lower ASYM on the previous day $(48.2 \pm 3.6 \%$ versus $50.0 \pm 4.7 \%, \mathrm{p}=0.087)$ and prior induction $(49.5 \%$ [47.4;51.5] versus 50.6\% [49.1;54.2], $\mathrm{p}=0.081)$ as compared to patients who never developed POD (Figure 2). At other time points, we observed no ASYM difference between the groups. ASYM was not a predictor of POD, since ROC analysis indicated an AUC of 0.63 , which was not significantly different from $0.5(p=0.08$, Figure 1 , Table 4$)$

\section{EEG burst suppression}

During the ICU stay, we observed no difference in BSR between patients who developed delirium and those who did not. However during surgery, a significantly $(\mathrm{p}=0.028)$ higher BSR was observed in patients who subsequently developed POD $(\mathrm{BSR}=1.24 \%$, IQR $[0.30 ; 3.34])$ as compared to those who did not $(\mathrm{BSR}=0.44 \%$, IQR $[0.06 ; 2.00])$. A significant difference in BSR between the groups was also observed for the time period between intubation and onset of cardiopulmonary bypass (Table 5 ). This phenomenon occurred on the left as well as the right side (Figure 3). Although burst suppression was observed during surgery, the BSR itself was low $(<10 \%)$.

During surgery, patients who later on developed delirium spent significantly $(\mathrm{p}=0.018)$ more time (107 minutes, IQR [47;170]) in a state of burst suppression than non-delirious patients (44 minutes, IQR [11;120], Table 6). ROC analysis identified BS duration as well as BS ratio as significant predictors of POD with an area under the curve of 0.73 and

Table 3 Intraoperative parameters

\begin{tabular}{|c|c|c|c|c|}
\hline & & \multicolumn{2}{|l|}{ Patients } & \multirow[t]{2}{*}{$\mathrm{p}$-value } \\
\hline & & With delirium & Without delirium & \\
\hline Duration of surgery & {$[\mathrm{min}]$} & $321 \pm 69$ & $326 \pm 74$ & 0.768 \\
\hline CPB time & {$[\mathrm{min}]$} & $131 \pm 33$ & $129 \pm 35$ & 0.786 \\
\hline \multicolumn{5}{|l|}{ Anaesthetic agents } \\
\hline Sufentanil (total dose) & {$[\mu \mathrm{g}]$} & $406 \pm 97$ & $417 \pm 106$ & 0.679 \\
\hline Isoflurane (average) & [etVol \%] & $0.7 \pm 0.1$ & $0.7 \pm 0.1$ & 0.679 \\
\hline \multicolumn{5}{|c|}{ Average arterial blood pressure } \\
\hline Intraoperatively & {$[\mathrm{mmHg}]$} & $87.8 \pm 7.0$ & $86.6 \pm 5.6$ & 0.464 \\
\hline During CPB & {$[\mathrm{mmHg}]$} & $65.1 \pm 5.4$ & $62.7 \pm 6.1$ & 0.125 \\
\hline Average body temperature & {$\left[{ }^{\circ} \mathrm{C}\right]$} & $35.6 \pm 0.4$ & $35.6 \pm 0.4$ & 0.515 \\
\hline
\end{tabular}

Data are displayed as mean \pm std dev. CPB = cardiopulmonary bypass. No significant differences between the groups were observed for any of the above mentioned parameters. 


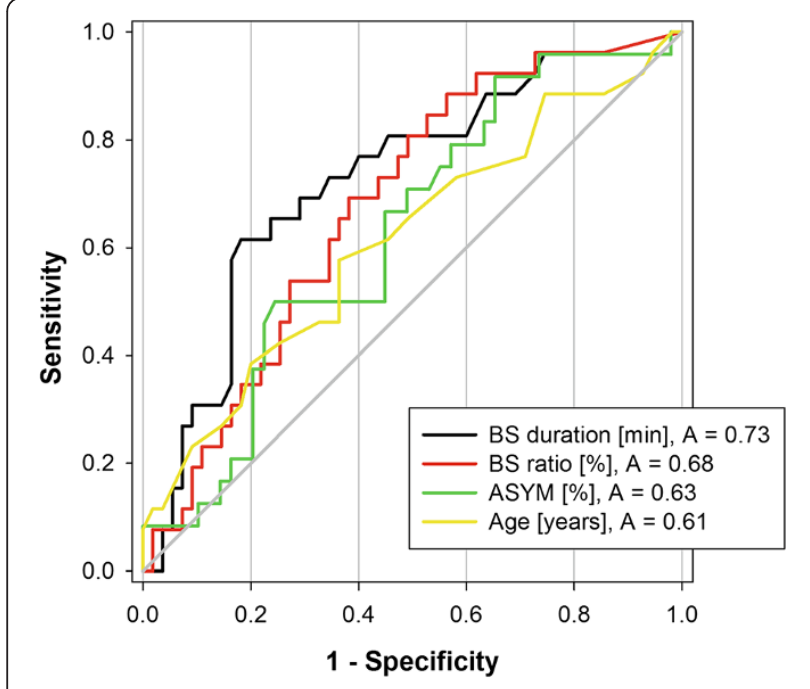

Figure 1 Receiver operating characteristic (ROC) analysis of four parameters to predict postoperative delirium. Burst suppression (BS) was assessed on the right side during the period from induction of anaesthesia until the onset of cardiopulmonary bypass. The area under the curve $(A)$ for the parameters BS duration and BS ratio was significantly ( $p=0.001$ and 0.009 respectively) different from 0.5 , whereas the area under the curve for age and ASYM was not. $\mathrm{ASYM}=\mathrm{EEG}$ asymmetry.

0.68 , respectively (Figure 1, Table 4). For cut-off values of 47 minutes BS duration and 5\% BS ratio, sensitivity, specificity and predictive value are shown in Table 4.

\section{Discussion}

\section{Asymmetry}

In our study, we applied bilateral BIS monitoring to investigate EEG asymmetry in the perioperative period of cardiac surgery. Contrary to the reported EEG lateralization during POD [11], patients with delirium did not show an altered asymmetry postoperatively in our study. Presumably, such lateralization phenomena might only be detected applying dedicated techniques such as Low Resolution Electromagnetic Tomography [25] or intracranial EEG recordings, but not using a single or dual channel EEG analyzer such as the BIS monitor, which analyses superficial and local electrical activity percutaneously.
However, during the preoperative period (that is, on the previous day as well as prior to induction), we observed a trend towards a lower ASYM (more EEG power on the right side) in patients that later on developed POD. This tendency has not been reported before and requires further investigation. So far, we have no explanation why this difference in ASYM between the delirious and the non-delirious group vanishes as soon as anaesthesia is induced. In addition, we observed an increase in ASYM from the previous day to the period prior induction, which might be related to the premedication (midazolam) given one hour before induction.

\section{Burst suppression}

In our study, patients who later developed POD, exhibited a significantly higher BSR during surgery and remained significantly longer in a state of $\mathrm{BSR}>0$ as compared to patients who did not develop POD. A possible explanation would be that "excessive" anaesthetic depth, as indicated by the occurrence of burst suppression, might have contributed to the development of POD and an increase in long-term mortality in our study. In fact, high doses of anaesthetics induce a burst suppression EEG, [26-28] and the cumulative duration of "deep" anaesthesia (specifically a BIS $<45$ ) has been shown to be associated with an increased long-term mortality [29-32]. In addition, Watson et al. reported a two-fold 6-month mortality in critically ill patients with a BSR $>0$ as compared to patients without burst suppression [15]. However, the anaesthetic depth in our study (median BIS $=41.3$ and 44.2 on the left and right side, respectively) was within the recommended range (BIS between 40 and 60) [33] and comparable with other studies of cardiac surgery: For instance, Kertai et al. reported an average intraoperative BIS of $40 \pm 7.2$ in 460 patients [29]. Moreover, the BIS during surgery did not differ in our study between patients who eventually developed POD and those who did not.

High doses of anaesthetics are associated with a high BSR, and above a BSR threshold of $40 \%$, the BIS is directly and linearly related to the BSR according to the formula $\mathrm{BIS}=50-\mathrm{BSR} / 2$ [26]. In contrast, the BSR values in our study were low $(<10 \%)$ and in a range, where the BIS remains unaffected. Therefore, it is unlikely that excessive

Table 4 Receiver operator characteristic (ROC) analysis regarding the ability of four parameters to predictor postoperative delirium

\begin{tabular}{|c|c|c|c|c|c|c|c|}
\hline Parameter & Area under the curve & p-value & Cut-off & Sensitivity & Specificity & Positive predictive value & Negative predictive value \\
\hline Burst suppression duration & 0.73 & 0.001 & 47 min. & 0.58 & 0.84 & 0.63 & 0.81 \\
\hline Burst suppression ratio & 0.68 & 0.009 & $5 \%$ & 0.23 & 0.89 & 0.50 & 0.71 \\
\hline ASYM & 0.63 & 0.08 & & & & & \\
\hline Age & 0.61 & 0.10 & & & & & \\
\hline
\end{tabular}

Burst suppression values for duration and ratio were obtained from the right forehead during the period from induction of anaesthesia until the onset of cardiopulmonary bypass. No cut-off data or predictive values were calculated for the parameters age and ASYM, since the area under their curve was nonsignificantly $(p>0.05)$ different from 0.5 (diagonal line). 


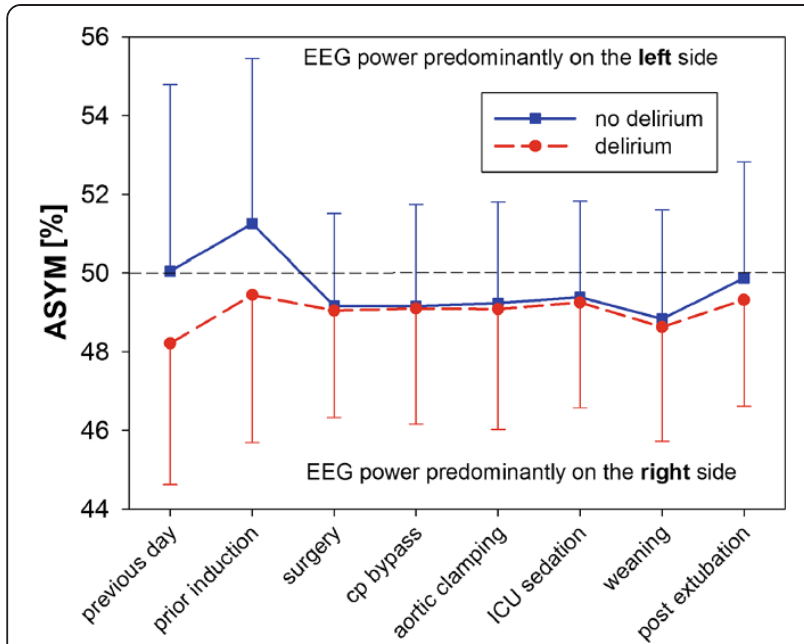

Figure 2 The time course of the EEG-asymmetry (ASYM) in comparison between delirious and non-delirious patients. An ASYM of less or more than 50\% indicates more EEG power on the right or left hemisphere, respectively. Cp bypass $=$ cardiopulmonary bypass. Data $=$ mean \pm std dev.

anaesthetic depth was the cause for the low BSR values observed in our study. A mean intraoperative BSR of 7.1 and $8.8 \%$ was observed by Radtke et al. [34] during noncardiac surgery in a BIS guided and a BIS blinded group, respectively, indicating that low BSR values occur during both cardiac and non-cardiac surgery. In fact, higher BSR values $(>10 \%)$ were observed by Besch et al. in $8.8 \%$ of patients undergoing noncardiac surgery [35]. They identified an age $>60$ years, history of coronary artery disease and male gender as independent factors associated with a BSR $>10 \%$, which are characteristics also present in the majority of our patients.

In general, an EEG with a high BSR indicates coma due to excessive anaesthetic depth [26] or other etiologies (hypoxia, intoxication, hypothermia, encephalopathy) [36]. However, the pathophysiology and clinical impact of a "low BSR EEG" remains unknown [35]. We speculate that it may indicate an increased sensitivity of the brain to the inflammatory response triggered by surgery. Alternatively, the occurrence of burst suppression at typical anaesthetic doses

Table 5 Burst Suppression Ratio during surgery

\begin{tabular}{|c|c|c|c|}
\hline \multirow[b]{2}{*}{ Group } & \multicolumn{3}{|l|}{ Patients } \\
\hline & With delirium & Without delirium & p-value \\
\hline \multicolumn{4}{|c|}{ Averaged over the entire period of surgery } \\
\hline Left BSR & $1.4 \%[0.3 ; 5.3]$ & $0.5 \%[0.1 ; 2.3]$ & 0.032 \\
\hline Right BSR & $0.7 \%[0.2 ; 2.9]$ & $0.4 \%[0.04 ; 1.3]$ & 0.021 \\
\hline \multicolumn{4}{|c|}{$\begin{array}{l}\text { Averaged over the period from intubation to onset of } \\
\text { cardiopulmonary bypass }\end{array}$} \\
\hline Left BSR & $1.8 \%[0.3 ; 4.9]$ & $0.5 \%[0.05 ; 2.5]$ & 0.035 \\
\hline Right BSR & $1.3 \%[0.4 ; 4.5]$ & $0.3 \%[0.02 ; 2.7]$ & 0.010 \\
\hline
\end{tabular}

All data are shown as median and interquartile range.

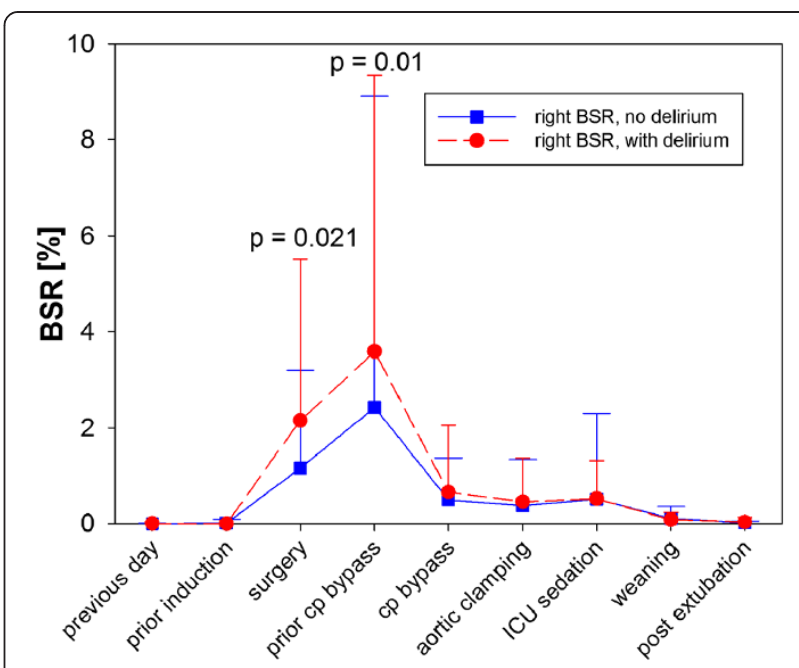

Figure 3 The Burst Suppression Ratio (BSR) as observed on the right side during the pre-, intra- and postoperative period. Data obtained in delirious or non-delirious patients are illustrated in red and blue, respectively. $\mathrm{Cp}$ bypass $=$ cardiopulmonary bypass. Data $=$ mean \pm std dev.

may identify patients with unusual anaesthetic sensitivity, in whom a "normal" anaesthetic dose is in fact a relative overdose [5]. This hypothesis is supported by Whitlock et al., who identified low average anaesthetic dose as an independent predictor of delirium [5]. Hence studies, which aim at reducing a low BSR EEG are required.

\section{Delirium}

The incidence of POD in our study (32\%) is within the reported range from 14 to 51\% [3-5]. Patients who developed POD experienced a higher long-term mortality than nondelirious patients in our study, which confirms earlier reports $[14,37,38]$.

\section{Limitations}

One limitation of our study is due to its design as an observational study. It remains unknown whether an intervention

Table 6 Time spent in a state of burst suppression, i.e. a BSR $>0 \%$

\begin{tabular}{|c|c|c|c|}
\hline \multirow[b]{2}{*}{ Group } & \multicolumn{3}{|l|}{ Patients } \\
\hline & With delirium & Without delirium & $p$-value \\
\hline \multicolumn{4}{|c|}{$\begin{array}{l}\text { Duration of burst suppression as obtained during the entire } \\
\text { period of surgery }\end{array}$} \\
\hline Left side & $131 \min [50 ; 183]$ & $48 \min [13 ; 127]$ & 0.034 \\
\hline Right side & $85 \min [46 ; 142]$ & $35 \min [7 ; 89]$ & 0.009 \\
\hline
\end{tabular}

Duration of burst suppression from intubation to onset of cardiopulmonary bypass

\begin{tabular}{llll} 
Left side & $59 \min [17 ; 77]$ & $20 \min [3 ; 58]$ & 0.008 \\
Right side & $53 \mathrm{~min}[18 ; 77]$ & $13 \mathrm{~min}[2 ; 37]$ & 0.001 \\
\hline
\end{tabular}

All data are shown as median and interquartile range. 
would have been effective to reduce or avoid burst suppression during surgery, and whether this would have reduced the incidence of delirium. Another limitation is the exclusion of patients under 60 years of age. It remains unknown whether our results could be extended to younger patients, especially since an age $>60$ years has been identified by Besch et al. [35] as a risk factor for the occurrence of a burst suppression EEG during anaesthesia.

\section{Conclusions}

The intraoperative assessment of the Burst Suppression Ratio may help to identify patients at risk for POD following cardiac surgery. So far it remains unknown, whether there is a causal relation or rather an association between BSR and the development of POD. Hence, further studies are required to clarify this question. We deem excessive depth of anaesthesia unlikely to be the underlying reason for the occurrence of burst suppression in our study, since the BIS was within the recommended range and the observed BSR was low. However, the clinical significance of such low BSR values is unknown and requires further investigations.

\section{Abbreviations}

ADCS-MCI-ADL: Alzheimer's Disease Cooperative Study Mild Cognitive Impairment Activities of Daily Living; ADL: Activity of daily living; ASYM: Asymmetry of the electroencephalogram; BIS: Bispectral Index ${ }^{\oplus}$; BSR: Burst suppression ratio; CABG: Coronary artery bypass grafting; CAM-ICU: Confusion assessment method for intensive care units; CPB: Cardiopulmonary bypass; EEG: Electroencephalogram; ICU: Intensive care unit; IQR: Interquartile range; MAC: Minimal alveolar concentration; POD: Postoperative delirium; ROC: Receiver operating characteristic.

\section{Competing interests}

MS and RKE have received honoraria for lectures from Covidien Germany (Neustadt/Donau, Germany). The other authors declare that they have no competing interests.

\section{Authors' contributions}

MS designed the study, acquired the funding, drafted the manuscript and participated in the data collection, analysis and interpretation. AD recruited the patients, collected the data and participated in data analysis and interpretation as well as in drafting the manuscript. RKE participated in data collection as well as manuscript drafting. GB participated in planning the study and interpreting the data. CP participated in the data analysis and interpretation. UG participated in designing the study, interpretation of data, as well as manuscript drafting. All authors read and approved the final manuscript.

\section{Acknowledgments}

The support of Prof. Dr. Armin Welz, Ingo Heinze, Beate Tuschy and her CRNSteam as well as of Alexander von Scholten and his nursing team of the cardiac surgery intensive care unit is gratefully acknowledged. Data presented here are part of the doctoral thesis of AD.

This study was funded in part by a grant of Covidien AG (Neuhausen, Switzerland) to MS. The sponsor had no influence on study design, study conductance, data analysis, preparation of the manuscript and the decision to submit the manuscript for publication.

\section{Author details}

${ }^{1}$ Department of Anaesthesiology and Intensive Care Medicine, University of Bonn, Bonn, Germany. ${ }^{2}$ Department of Neurology and Psychiatry, LVR-Clinic, Bonn, Germany.

Received: 17 July 2014 Accepted: 22 April 2015

Published online: 28 April 2015

\section{References}

1. American Psychiatric Association. Diagnostic and Statistical Manual of Mental Disorders - DSM-IV-TR. 4th ed. Washington DC: American Psychiatric Association; 2000.

2. Guenther U, Theuerkauf N, Frommann I, Brimmers K, Malik R, Stori S, et al. Predisposing and precipitating factors of delirium after cardiac surgery: a prospective observational cohort study. Ann Surg. 2013;257(6):1160-7.

3. Mu DL, Wang DX, Li LH, Shan GJ, Li J, Yu QJ, et al. High serum cortisol level is associated with increased risk of delirium after coronary artery bypass graft surgery: a prospective cohort study. Crit Care. 2010;14(6):R238.

4. van der Mast RC, van den Broek WW, Fekkes D, Pepplinkhuizen L, Habbema JD. Incidence of and preoperative predictors for delirium after cardiac surgery. J Psychosom Res. 1999;46(5):479-83.

5. Whitlock EL, Torres BA, Lin N, Helsten DL, Nadelson MR, Mashour GA, et al. Postoperative delirium in a substudy of cardiothoracic surgical patients in the BAG-RECALL clinical trial. Anesth Analg. 2014;118(4):809-17.

6. Ely EW, Shintani A, Truman B, Speroff T, Gordon SM, Harrell Jr FE, et al. Delirium as a predictor of mortality in mechanically ventilated patients in the intensive care unit. JAMA. 2004;291(14):1753-62.

7. Guenther U, Radtke FM. Delirium in the postanaesthesia period. Curr Opin Anaesthesiol. 2011;24(6):670-5.

8. Koponen H, Partanen J, Paakkonen A, Mattila E, Riekkinen PJ. EEG spectral analysis in delirium. J Neurol Neurosurg Psychiatry. 1989;52(8):980-5.

9. Plaschke K, Hill H, Engelhardt R, Thomas C, von Haken R, Scholz M, et al. EEG changes and serum anticholinergic activity measured in patients with delirium in the intensive care unit. Anaesthesia. 2007;62(12):1217-23.

10. Thomas C, Hestermann U, Kopitz J, Plaschke K, Oster P, Driessen M, et al. Serum anticholinergic activity and cerebral cholinergic dysfunction: an EEG study in frail elderly with and without delirium. BMC Neurosci. 2008;9:86

11. Reischies FM, Neuhaus AH, Hansen ML, Mientus S, Mulert C, Gallinat J. Electrophysiological and neuropsychological analysis of a delirious state: the role of the anterior cingulate gyrus. Psychiatry Res. 2005;138(2):171-81.

12. Soehle M, Ellerkmann RK, Grube M, Kuech M, Wirz S, Hoeft A, et al. Comparison between bispectral index and patient state index as measures of the electroencephalographic effects of sevoflurane. Anesthesiology. 2008;109(5):799-805.

13. Soehle M, Kuech M, Grube M, Wirz S, Kreuer S, Hoeft A, et al. Patient state index vs bispectral index as measures of the electroencephalographic effects of propofol. Br J Anaesth. 2010;105(2):172-8.

14. Gottesman RF, Grega MA, Bailey MM, Pham LD, Zeger SL, Baumgartner WA, et al. Delirium after coronary artery bypass graft surgery and late mortality. Ann Neurol. 2010;67(3):338-44.

15. Watson PL, Shintani AK, Tyson R, Pandharipande PP, Pun BT, Ely EW. Presence of electroencephalogram burst suppression in sedated, critically ill patients is associated with increased mortality. Crit Care Med. 2008;36(12):3171-7.

16. Kelley SD. Monitoring consciousness using the Bispectral Index during anesthesia. 2nd ed. Boulder, CO: Covidien; 2010.

17. Aspect Medical Systems: BIS VISTA Monitoring System - Bilateral Monitoring Addendum. Newton, MA 2010.

18. Aspect Medical Systems: BIS VISTA Monitoring System - Operating Manual. Newton, MA 2008.

19. Rampil IJ. A primer for EEG signal processing in anesthesia. Anesthesiology. 1998;89(4):980-1002.

20. Ely EW, Inouye SK, Bernard GR, Gordon S, Francis J, May L, et al. Delirium in mechanically ventilated patients: validity and reliability of the confusion assessment method for the intensive care unit (CAM-ICU). JAMA. 2001;286(21):2703-10

21. Guenther U, Popp J, Koecher L, Muders T, Wrigge H, Ely EW, et al. Validity and reliability of the CAM-ICU Flowsheet to diagnose delirium in surgical ICU patients. J Crit Care. 2010;25(1):144-51

22. Sessler CN, Gosnell MS, Grap MJ, Brophy GM, O'Neal PV, Keane KA, et al. The Richmond Agitation-Sedation Scale: validity and reliability in adult intensive care unit patients. Am J Respir Crit Care Med. 2002;166(10):1338-44.

23. Pandharipande P, Cotton BA, Shintani A, Thompson J, Costabile S, Truman Pun $B$, et al. Motoric subtypes of delirium in mechanically ventilated surgical and trauma intensive care unit patients. Intensive Care Med. 2007;33(10):1726-31.

24. Galasko D, Bennett D, Sano M, Ernesto C, Thomas R, Grundman M, et al. An inventory to assess activities of daily living for clinical trials in Alzheimer's disease. The Alzheimer's Disease Cooperative Study. Alzheimer Dis Assoc Disord. 1997;11 Suppl 2:S33-39. 
25. Pascual-Marqui RD, Michel CM, Lehmann D. Low resolution electromagnetic tomography: a new method for localizing electrical activity in the brain. Int J Psychophysiol. 1994;18(1):49-65.

26. Bruhn J, Bouillon TW, Shafer SL. Bispectral index (BIS) and burst suppression: revealing a part of the BIS algorithm. J Clin Monit Comput. 2000;16(8):593-6.

27. Hartikainen KM, Rorarius M, Perakyla JJ, Laippala PJ, Jantti V. Cortical reactivity during isoflurane burst-suppression anesthesia. Anesth Analg. 1995:81(6):1223-8.

28. Yoshitani K, Kawaguchi M, Takahashi M, Kitaguchi K, Furuya H. Plasma propofol concentration and EEG burst suppression ratio during normothermic cardiopulmonary bypass. Br J Anaesth. 2003;90(2):122-6.

29. Kertai MD, Pal N, Palanca BJ, Lin N, Searleman SA, Zhang L, et al. Association of perioperative risk factors and cumulative duration of low bispectral index with intermediate-term mortality after cardiac surgery in the B-Unaware Trial. Anesthesiology. 2010;112(5):1116-27.

30. Leslie K, Myles PS, Forbes A, Chan MT. The effect of bispectral index monitoring on long-term survival in the B-aware trial. Anesth Analg. 2010;110(3):816-22.

31. Lindholm ML, Traff S, Granath F, Greenwald SD, Ekbom A, Lennmarken C, et al. Mortality within 2 years after surgery in relation to low intraoperative bispectral index values and preexisting malignant disease. Anesth Analg. 2009;108(2):508-12.

32. Monk TG, Saini V, Weldon BC, Sigl JC. Anesthetic management and one-year mortality after noncardiac surgery. Anesth Analg. 2005;100(1):4-10.

33. Kelley SD. Monitoring level of consciousness during anesthesia and sedation. A clinician's guide to the Bispectral Index. Newton: Aspect Medical Systems, Inc.; 2003

34. Radtke FM, Franck M, Lendner J, Kruger S, Wernecke KD, Spies CD. Monitoring depth of anaesthesia in a randomized trial decreases the rate of postoperative delirium but not postoperative cognitive dysfunction. $\mathrm{Br} J$ Anaesth. 2013;110 Suppl 1:198-105.

35. Besch G, Liu N, Samain E, Pericard C, Boichut N, Mercier M, et al. Occurrence of and risk factors for electroencephalogram burst suppression during propofol-remifentanil anaesthesia. Br J Anaesth. 2011;107(5):749-56.

36. Brenner RP. The interpretation of the EEG in stupor and coma. Neurologist. 2005; 11(5):271-84.

37. Koster $\mathrm{S}$, Hensens AG, van der Palen J. The long-term cognitive and functional outcomes of postoperative delirium after cardiac surgery. Ann Thorac Surg. 2009:87(5):1469-74.

38. Loponen P, Luther M, Wistbacka JO, Nissinen J, Sintonen $\mathrm{H}$, Huhtala H, et al. Postoperative delirium and health related quality of life after coronary artery bypass grafting. Scand Cardiovasc J. 2008;42(5):337-44.

\section{Submit your next manuscript to BioMed Central and take full advantage of:}

- Convenient online submission

- Thorough peer review

- No space constraints or color figure charges

- Immediate publication on acceptance

- Inclusion in PubMed, CAS, Scopus and Google Scholar

- Research which is freely available for redistribution 